\title{
Mutations in rps $L$ that confer streptomycin resistance show pleiotropic effects on virulence and the production of a carbapenem antibiotic in Erwinia carotovora
}

Correspondence

George P. C. Salmond

gpcs@mole.bio.cam.ac.uk

Received 25 September 2009

Revised 6 December 2009

Accepted 24 December 2009

\author{
Anne M. L. Barnard, Natalie J. L. Simpson, Kathryn S. Lilley \\ and George P. C. Salmond \\ Department of Biochemistry, University of Cambridge, Tennis Court Road, \\ Cambridge CB2 1OW, UK
}

\begin{abstract}
Spontaneous streptomycin-resistant derivatives of Erwinia carotovora subsp. carotovora strain ATTn10 were isolated. Sequencing of the rps $L$ locus (encoding the ribosomal protein S12) showed that each mutant was missense, with a single base change, resulting in the substitution of the wild-type lysine by arginine, threonine or asparagine at codon 43. Phenotypic analyses showed that the rpsL mutants could be segregated into two groups: K43R mutants showed reduced production of the $\beta$-lactam secondary metabolite 1-carbapen-2-em-3 carboxylic acid (Car), but little effect on exoenzyme production or virulence in potato tuber tests. By contrast, the $\mathrm{K} 43 \mathrm{~N}$ and $\mathrm{K} 43 \mathrm{~T}$ mutations were pleiotropic, resulting in reduced exoenzyme production and virulence, as well as diminished Car production. The effect on Car production was due to reduced transcription of the quorum-sensing-dependent car biosynthetic genes. The effects of $\mathrm{K} 43 \mathrm{~N}$ and K43T mutations on Car production were partially alleviated by provision of an excess of the quorum-sensing signalling molecule $\mathrm{N}$-(3-oxohexanoyl)-L-homoserine lactone. Finally, a proteomic analysis of the K43T mutant indicated that the abundance of a subset of intracellular proteins was affected by this $r p s L$ mutation.
\end{abstract}

\section{INTRODUCTION}

Erwinia carotovora subsp. carotovora $(E c c)$ is a Gramnegative phytopathogen which causes soft rot disease of a variety of plants hosts (including potato), by the production of an arsenal of plant cell wall degrading exoenzymes, such as pectate lyases, cellulases and proteases (reviewed by Toth \& Birch, 2005). In addition, some strains of Ecc, including strain ATCC 39048 and its derivatives, ATTn10 and MS1, produce the secondary metabolite antibiotic 1-carbapen-2-em-3-carboxylic acid (Car; Bainton et al., 1992a, b). Production of exoezymes and Car is under the control of the quorum-sensing (QS) system in $E c c$, such that these factors are only produced during the lateexponential and stationary phases of growth in laboratory culture. The QS signal is $N$-(3-oxohexanoyl)-L-homoserine lactone (3-oxo-C6-HSL); its synthesis is catalysed by the CarI synthase (Bainton et al., 1992a, b; Jones et al., 1993). QS signals are normally sensed by members of the LuxR family of transcriptional regulators (reviewed by Whitehead et al., 2001; Barnard \& Salmond, 2005; Waters

Abbreviations: Car, 1-carbapen-2-em-3 carboxylic acid; DiGE, difference gel electrophoresis; Eca, Erwinia carotovora subsp. atroseptica; Ecc, Erwinia carotovora subsp. carotovora; 3-oxo-C6-HSL, N-(3-oxohexanoyl)-L-homoserine lactone; OS, quorum sensing.
\& Bassler, 2005). In Ecc strain ATTn10, three $\operatorname{luxR}$ homologues have been identified. The expR gene is located adjacent to carI in the genome; however, the function of ExpR is unclear since an $\operatorname{expR}$ mutant appears to have no obvious phenotype in this strain (Rivet, 1998). Exoenzymes are controlled via the LuxR homologue VirR (Burr et al., 2006), while Car production is activated by the third LuxR homologue, CarR (McGowan et al., 1995, 2005). In addition to these QS inputs, Car and exoenzyme production are coordinately regulated by the Hor global activator, which is a member of the SlyA family of transcriptional regulators (Thomson et al., 1997; McGowan et al., 2005).

While carrying out a screen for transposon-induced Ecc mutants showing defects in carbapenem production, we isolated two streptomycin-resistant derivatives of strain ATTn10 that showed reduced Car production, but did not appear to carry the transposon. The streptomycin resistance appeared to be a spontaneous mutation in these isolates, and we hypothesized that the impact on Car production may have been due to the same spontaneous mutation. We therefore carried out further experiments to isolate more spontaneous streptomycin-resistant mutants, without using transposon mutagenesis. Here, we describe 
the nature of the corresponding mutations and their pleiotropic effects on Car antibiotic production, plant cell wall degrading enzymes, virulence and antibiotic resistance.

\section{METHODS}

Bacterial strains and culture conditions. All bacterial strains (Table 1) were cultured in LB medium ( $10 \mathrm{~g}$ tryptone, $5 \mathrm{~g}$ yeast extract, $5 \mathrm{~g} \mathrm{NaCl}$ per litre) unless otherwise stated. For agar plates, Bacto agar $(1.6 \%, \mathrm{w} / \mathrm{v})$ was added (or $0.7 \%, \mathrm{w} / \mathrm{v}$, for top agar layers) Growth temperatures were $30{ }^{\circ} \mathrm{C}$ for Ecc and $37{ }^{\circ} \mathrm{C}$ for Escherichia coli (E. coli) strains, unless otherwise stated. For isolation of streptomycin-resistant mutants, overnight cultures of strains ATTn10 or MS1 (a $\mathrm{Lac}^{-}$derivative of ATTn10) were used to inoculate $50 \mathrm{ml} \mathrm{LB}$ to a starting $\mathrm{OD}_{600}$ of 0.05 . Cultures were incubated for $24 \mathrm{~h}$ (at $30{ }^{\circ} \mathrm{C}$ in a shaking incubator set at 250 r.p.m.), before resuspension in $1 \mathrm{ml} \mathrm{LB}$ and plating in $100 \mu \mathrm{l}$ aliquots onto LB agar containing $100 \mu \mathrm{g}$ streptomycin $\mathrm{ml}^{-1}$.

DNA manipulations. Molecular biology techniques were performed as described by Sambrook et al. (1989). Enzymes were purchased from NEB or Invitrogen and used according to manufacturers' instructions. The Expand High Fidelity PCR system (Roche) was used in PCR. Oligonucleotides (Table 3) were purchased from SigmaGenosys. DNA sequencing was carried out by the DNA Sequencing Facility, Department of Biochemistry, University of Cambridge, UK.

$\phi \mathbf{K P}$-mediated generalized transduction. The Ecc-specific generalized transducing bacteriophage $\phi \mathrm{KP}$ was used to move chromosomal markers between Ecc strains, as described by Toth et al. (1993).

Antibiotic bioassay. Carbapenem antibiotic production was assayed via the inhibition of growth of a $\beta$-lactam-sensitive $E$. coli strain (ESS). A top agar layer seeded with ESS (100 $\mu$ l overnight culture was added to $100 \mathrm{ml} \mathrm{LB}$ medium containing $0.7 \%$, w/v, agar) was poured onto standard LB agar plates. Aliquots $(3 \mu \mathrm{l})$ of overnight $E c c$ cultures (normalized to an $\mathrm{OD}_{600}$ of 1.0 by dilution with $\mathrm{LB}$ medium) were spotted onto the ESS lawn. Alternatively, cup wells were made in the ESS lawn using a sterilized cork borer, and the wells filled with $80 \mu \mathrm{l}$ filter-sterilized (through a $0.2 \mu \mathrm{m}$ filter) Ecc culture supernatant. Car production was indicated by a zone of antibiosis of the ESS lawn around the test site, following overnight incubation at $30{ }^{\circ} \mathrm{C}$.

$\mathbf{N}$-Acylhomoserine lactone bioassay. Bioassay plates were prepared as described for the antibiotic bioassay, except that the top agar layer was seeded with the $\mathrm{N}$-acylhomoserine lactone sensor strain of Chromobacterium violaceum $\mathrm{CV} 026$ ( $1 \mathrm{ml}$ of an overnight culture was added to $100 \mathrm{ml} \mathrm{LB}$ medium containing $0.7 \%$, w/v, agar). Aliquots $(3 \mu \mathrm{l})$ of overnight $E c c$ cultures (normalized to an $\mathrm{OD}_{600}$ of 1.0 by dilution with LB medium) were spotted onto the CV026 lawn. $\mathrm{N}$-Acylhomoserine lactone production was indicated by a purple halo in the CV026 lawn around the test site, following overnight incubation at $30{ }^{\circ} \mathrm{C}$.

$\boldsymbol{\beta}$-Galactosidase assay. Samples $(1 \mathrm{ml})$ were taken at hourly intervals throughout growth of strains in LB medium. Cells were permeabilized by the addition of $50 \mu$ toluene followed by vortexing for $30 \mathrm{~s}$. The organic and aqueous phases were allowed to separate and $\beta$-galactosidase assays were performed using the (aqueous) lysate. The rate of colour development at $37{ }^{\circ} \mathrm{C}$, following the addition of ONPG, was measured using a Unicam He $\lambda$ ios $\alpha$ spectrophotometer set to measure absorbance at $420 \mathrm{~nm}$. Activities are expressed as arbitrary units and are proportional to the increase in $A_{420}$ per minute per constant cell density. Three replicates of each strain to be tested were cultured and assayed for $\beta$-galactosidase activity.

Pectate lyase, cellulase and protease agar plate assays. Aliquots $(5 \mu \mathrm{l})$ of overnight $E c c$ cultures (normalized to an $\mathrm{OD}_{600}$ of 1.0 by dilution with LB medium) were spotted onto pectate lyase assay plates $[1.6 \%(\mathrm{w} / \mathrm{v})$ Bacto agar, $0.1 \%(\mathrm{w} / \mathrm{v})$ yeast extract, $0.1 \%(\mathrm{w} / \mathrm{v})$ $\left(\mathrm{NH}_{4}\right)_{2} \mathrm{SO}_{4}, 1 \mathrm{mM} \mathrm{MgSO}, \quad 0.5 \% \quad(\mathrm{v} / \mathrm{v})$ glycerol, $0.5 \% \quad(\mathrm{w} / \mathrm{v})$ polygalacturonic acid, (sodium salt), $20 \%(\mathrm{v} / \mathrm{v})$ pel phosphate buffer (15 $\mathrm{g} \mathrm{Na}_{2} \mathrm{HPO}_{4}, 0.7 \mathrm{~g} \mathrm{NaH}_{2} \mathrm{PO}_{4} \cdot \mathrm{H}_{2} \mathrm{O}$ per litre, $\mathrm{pH}$ 8.0)], cellulase assay plates $[1.6 \%(\mathrm{w} / \mathrm{v})$ Bacto agar, $1 \%(\mathrm{w} / \mathrm{v})$ carboxymethylcellulose, $0.5 \%$ $(\mathrm{w} / \mathrm{v})$ yeast extract, $0.2 \%(\mathrm{v} / \mathrm{v})$ glycerol, $2 \%(\mathrm{v} / \mathrm{v}) 50 \times$ phosphate buffer $\left(350 \mathrm{~g} \mathrm{~K}_{2} \mathrm{HPO}_{4}, 100 \mathrm{~g} \mathrm{KH}_{2} \mathrm{PO}_{4}\right.$ per litre, $\mathrm{pH}$ 6.9-7.1), $0.1 \%$ (w/ v) $\left(\mathrm{NH}_{4}\right)_{2} \mathrm{SO}_{4}, 0.01 \%(\mathrm{w} / \mathrm{v}) \mathrm{MgSO}_{4}$ ] or protease assay plates [Oxoid nutrient agar, supplemented with $0.03 \%(\mathrm{w} / \mathrm{v})$ gelatin]. Exoenzyme production was visualized following overnight incubation at $30{ }^{\circ} \mathrm{C}$. Pectate lyase plates were flooded with $7.5 \%(w / v)$ copper acetate for $1 \mathrm{~h}$; positive colonies were identified by double, cream haloes on a translucent blue-green background. Cellulase plates were stained with $0.2 \%(\mathrm{w} / \mathrm{v})$ Congo red solution for $20 \mathrm{~min}$, bleached with $1 \mathrm{M} \mathrm{NaCl}$ for $15 \mathrm{~min}$, then stained with $1 \mathrm{M} \mathrm{HCl}$ for $5 \mathrm{~min}$; positive colonies were identified by orange-red haloes on a dark blue background. Protease plates were flooded with $4 \mathrm{M}\left(\mathrm{NH}_{4}\right)_{2} \mathrm{SO}_{4}$; positive colonies were identified by clear haloes on an opaque white background.

Potato tuber virulence assays. Potato tubers (Solanum tuberosum 'Maris Piper') were washed and surface-sterilized by immersion in $5 \%$ hypochlorite solution for $10 \mathrm{~min}$, followed by washing in running water. Overnight cultures of test strains were diluted in LB and approximately $10^{4}$ c.f.u. were injected into uniform holes which had been bored into the potato using a pipette tip. Each potato contained an inoculation site for the wild-type, in addition to those for the various mutants tested. The inoculation sites were sealed using silicon grease. Inoculated potatoes were wrapped in alternating layers of damp tissue paper and cling-film and incubated at $25{ }^{\circ} \mathrm{C}$. The mass of soft rot was determined at $24 \mathrm{~h}$ intervals.

Western blot analysis. Culture samples were harvested and resuspended in $100 \mathrm{mM}$ Tris, $\mathrm{pH} 8.0$, to an $\mathrm{OD}_{600}$ of 2.0. Following sonication, cracking buffer was added to $1 \times$ final concentration [ $5 \times$ cracking buffer was made up as follows: $1 \mathrm{ml} 0.5 \mathrm{M}$ Tris/ $\mathrm{HCl}$ (pH 6.8), $0.8 \mathrm{ml}$ glycerol, $1.6 \mathrm{ml} 10 \%$ (w/v) SDS, $0.4 \mathrm{ml}$ $\beta$-mercaptoethanol, $0.4 \mathrm{ml} 1 \%(\mathrm{w} / \mathrm{v})$ bromophenol blue, $3.8 \mathrm{ml}$ $\mathrm{H}_{2} \mathrm{O}$ ]. Samples were boiled for $5 \mathrm{~min}$ prior to loading equivalent amounts of total protein on a $12 \%$ SDS-polyacrylamide gel. SDSPAGE was performed as described by Laemmli (1970). After electrotransfer to a PVDF filter, CarA protein was immunodetected with CarA-specific polyclonal antibodies and chemiluminescent reagents $(\mathrm{ECL}+$ Plus, Amersham).

2D-difference gel electrophoresis (2D-DiGE). Four overnight cultures of each of strains MS1 and M3 were used to inoculate $50 \mathrm{ml}$ $\mathrm{LB}$ in $500 \mathrm{ml}$ capacity flasks, to a starting $\mathrm{OD}_{600}$ of 0.05 . Cultures were grown at $30{ }^{\circ} \mathrm{C}, 250$ r.p.m., for $16 \mathrm{~h}$. Intracellular protein was harvested from $20 \mathrm{ml}$ of culture by centrifuging at $2739 \mathrm{~g}$ at $4{ }^{\circ} \mathrm{C}$ for $10 \mathrm{~min}$ and resuspending in CHAPS/urea lysis buffer containing $1 \times$ protease inhibitor. Each sample was sonicated $(3 \times 10 \mathrm{~s}$ bursts, keeping samples on ice). The cell debris was pelleted by centrifugation $\left(2220 \mathrm{~g}, 15 \mathrm{~min}, 4{ }^{\circ} \mathrm{C}\right)$. The supernatant was taken off into a fresh tube and centrifuged again in a benchtop microfuge (max speed, $10 \mathrm{~min}, 4^{\circ} \mathrm{C}$ ). The supernatant was removed into a fresh tube and stored in aliquots at $-20{ }^{\circ} \mathrm{C}$. Protein concentration was estimated using the Bio-Rad DC kit. Protein samples were reciprocally labelled with fluorescent cyanine dyes, Cy3 and Cy5 (GE Healthcare), as described by Coulthurst et al. (2006). A pool of all samples was labelled with Cy2 to use as a standard for each gel. 2D-PAGE separation of the proteins was also carried out as described by 
Table 1. Bacterial strains, plasmids and bacteriophage

\begin{tabular}{|c|c|c|}
\hline Strain & Genotype/phenotype & Reference \\
\hline \multicolumn{3}{|l|}{$E c c$} \\
\hline ATTn10 & Wild-type & McGowan et al. (1996) \\
\hline MS1 & $\mathrm{Lac}^{-}$derivative of ATTn10 & Holden et al. (1998) \\
\hline $\mathrm{AB} 1$ & $r p s L \mathrm{~K} 43 \mathrm{~T}, \mathrm{Stm}^{\mathrm{R}}$ derivative of ATTn10 & This study \\
\hline $\mathrm{AB} 2$ & $r p s L \mathrm{~K} 43 \mathrm{~T}, \mathrm{Stm}^{\mathrm{R}}$ derivative of ATTn10 & This study \\
\hline M1 & $r p s L \mathrm{~K} 43 \mathrm{~N}, \mathrm{Stm}^{\mathrm{R}}$ derivative of MS1 & This study \\
\hline M2 & rpsL K43R, Stm $^{\mathrm{R}}$ derivative of MS1 & This study \\
\hline M3 & $r p s L \mathrm{~K} 43 \mathrm{~T}, \mathrm{Stm}^{\mathrm{R}}$ derivative of MS1 & This study \\
\hline M4 & $r p s L \mathrm{~K} 43 \mathrm{~T}, \mathrm{Stm}^{\mathrm{R}}$ derivative of MS1 & This study \\
\hline M6 & rpsL K43T, Stm $^{\mathrm{R}}$ derivative of MS1 & This study \\
\hline M7 & rpsL K43R, Stm $^{\mathrm{R}}$ derivative of MS1 & This study \\
\hline M8 & rpsL K43R, Stm $^{\mathrm{R}}$ derivative of MS1 & This study \\
\hline M9 & $r p s L \mathrm{~K} 43 \mathrm{~N}, \mathrm{Stm}^{\mathrm{R}}$ derivative of MS1 & This study \\
\hline M10 & $r p s L \mathrm{~K} 43 \mathrm{R}, \mathrm{Stm}^{\mathrm{R}}$ derivative of MS1 & This study \\
\hline M11 & $r p s L \mathrm{~K} 43 \mathrm{~T}, \mathrm{Stm}^{\mathrm{R}}$ derivative of MS1 & This study \\
\hline M12 & rpsL K43T, Stm $^{\mathrm{R}}$ derivative of MS1 & This study \\
\hline M13 & rpsL K43R, Stm $^{\mathrm{R}}$ derivative of MS1 & This study \\
\hline M14 & rpsL K43R, Stm $^{\mathrm{R}}$ derivative of MS1 & This study \\
\hline M15 & rpsL K43R, Stm $^{\mathrm{R}}$ derivative of MS1 & This study \\
\hline M16 & $r p s L \mathrm{~K} 43 \mathrm{R}, \mathrm{Stm}^{\mathrm{R}}$ derivative of MS1 & This study \\
\hline GB7 & $\operatorname{car} A:: \operatorname{Tn} p h o A-2^{\prime}$ derivative of MS1, Tet ${ }^{\mathrm{R}}$ & Thomson et al. (1997) \\
\hline $7 \mathrm{M} 1$ & $\begin{array}{l}\text { carA:: TnphoA-2', rpsL K43N derivative of strain M1, } \\
\text { generated by transduction using } \phi \mathrm{KP} \text { grown on GB7 }\end{array}$ & This study \\
\hline $7 \mathrm{M} 3$ & $\begin{array}{l}\text { carA:: } \operatorname{Tn} p h o A-2^{\prime}, r p s L \mathrm{~K} 43 \mathrm{~T} \text { derivative of strain } \mathrm{M} 3 \text {, } \\
\text { generated by transduction using } \phi \mathrm{KP} \text { grown on GB7 }\end{array}$ & This study \\
\hline $7 \mathrm{M} 7$ & $\begin{array}{l}\text { carA:: TnphoA-2', rpsL K43R derivative of strain M7, } \\
\text { generated by transduction using } \phi \mathrm{KP} \text { grown on GB7 }\end{array}$ & This study \\
\hline GB3 & carR:: TnphoA-2' derivative of MS1, Tet ${ }^{\mathrm{R}}$ & Thomson et al. (1997) \\
\hline M1GB3 & $\begin{array}{l}\text { carR:: } \operatorname{Tn} p h o A-2^{\prime}, r p s L \mathrm{~K} 43 \mathrm{~N} \text { derivative of strain } \mathrm{M} 1 \text {, } \\
\text { generated by transduction using } \phi \mathrm{KP} \text { grown on } \mathrm{GB} 3\end{array}$ & This study \\
\hline M3GB3 & $\begin{array}{l}\text { carR:: TnphoA-2', rpsL K43T derivative of strain M3, } \\
\text { generated by transduction using } \phi \mathrm{KP} \text { grown on GB3 }\end{array}$ & This study \\
\hline M7GB7 & $\begin{array}{l}\text { carR:: TnphoA-2', rpsL K43R derivative of strain M7, } \\
\text { generated by transduction using } \phi \mathrm{KP} \text { grown on GB3 }\end{array}$ & This study \\
\hline DET107 & hor: : lacZ derivative of MS1 & McGowan et al. (2005) \\
\hline DETM1 & hor:: lacZ, rpsL K43N derivative of DET107 & This study \\
\hline DETM3 & hor: : lacZ, rpsL K43T derivative of DET107 & This study \\
\hline DETM7 & hor: : lacZ, rpsL K43R derivative of DET107 & This study \\
\hline PRTX1 & carI: $: \operatorname{Tn} p h o A-2^{\prime}$ derivative of $\mathrm{MS} 1, \mathrm{Tet}^{\mathrm{R}}$ & Cox et al. (1998) \\
\hline PRTM1 & $\begin{array}{l}\text { rpsL K } 43 \mathrm{~N} \text {, carI: : TnphoA-2' derivative of strain } \mathrm{M} 1 \text {, } \\
\text { generated by transduction using } \phi \mathrm{KP} \text { grown on strain PRTX1 }\end{array}$ & This study \\
\hline PRTM3 & $\begin{array}{l}\text { rpsL K43T, carI: : TnphoA-2' derivative of strain } \mathrm{M} 3 \text {, } \\
\text { generated by transduction using } \phi \mathrm{KP} \text { grown on strain PRTX1 }\end{array}$ & This study \\
\hline PRTM7 & $\begin{array}{l}\text { rpsL K43R, carI: : Tn } p h o A-2^{\prime} \text { derivative of strain } \mathrm{M} 7 \\
\text { generated by transduction using } \phi \mathrm{KP} \text { grown on strain PRTX1 }\end{array}$ & This study \\
\hline SM1 & $\Delta c a r A$ & McGowan et al. (1997) \\
\hline \multicolumn{3}{|l|}{ E. coli } \\
\hline ESS & $\beta$-Lactam sensitive & McGowan et al. (1997) \\
\hline \multicolumn{3}{|l|}{ Plasmids } \\
\hline pQE-80L & Qiagen expression vector; $\operatorname{lacl}^{\mathrm{q}}, \mathrm{Amp}^{\mathrm{R}}$ & Qiagen \\
\hline pAB536 & $\begin{array}{l}\text { Derivative of } \mathrm{pQE}-80 \mathrm{~L} \text { with } \mathrm{Amp}^{\mathrm{R}} \text { gene disrupted by insertion of a } \mathrm{Kan}^{\mathrm{R}} \\
\text { cassette (PCR-amplified from pACYC177) into the unique } X m n \mathrm{I} \text { site }\end{array}$ & This study \\
\hline pAB539 & Derivative of pAB536 carrying $r p s L$ from $E c c$ strain MS1 & This study \\
\hline pACYC177 & Medium-copy cloning vector, $\mathrm{Amp}^{\mathrm{R}} \operatorname{Kan}^{\mathrm{R}}$ & NEB \\
\hline \multicolumn{3}{|c|}{ Bacteriophage } \\
\hline$\phi \mathrm{KP}$ & Generalized transducing phage for Ecc strain ATCC 39048 and its derivatives & Toth et al. (1993) \\
\hline
\end{tabular}


Table 2. Car phenotypes and genotypes of $r p s L$ mutants

\begin{tabular}{|lllll|}
\hline Strain & Car phenotype & Codon $\mathbf{4 3}$ sequence & Amino acid at codon $\mathbf{4 3}$ & Amino acid change \\
\hline MS1, ATTn10 & Wild-type & AAA & Lysine & K43 \\
AB1, AB2, M3, M4, M6, M11, M12 & Car $^{-}$ & ACA & Threonine & K43T \\
M1, M9 & $\mathrm{Car}^{-}$ & AA $\underline{C}$ & Asparagine & K43N \\
M2, M7, M8, M10, M13, M14, M15, M16 & Car reduced & A $\underline{A}$ & Arginine & K43R \\
\hline
\end{tabular}

Coulthurst et al. (2006). Proteins were separated over $\mathrm{pH} 4-7$ in the first dimension followed by SDS-PAGE on a $12 \%$ gel for the second dimension. Labelled proteins were visualized using a Typhoon 9410 imager (Amersham Biosciences). Gels were then fixed and stained using colloidal Coomassie blue. The images were subjected to a biological variance analysis (BVA) using DeCyder BVA software (GE Healthcare). Protein spots which were increased in abundance by more than 1.5-fold and with a corresponding $P$-value $<0.01$ from univariate statistical analysis using a Student's $t$-test, in the wild-type or in the mutant, were identified. For mass spectrometry identification of protein spots, the spots were cut out from Coomassie-stained gels, digested with trypsin and then analysed by LC-MS/MS using a Q-TOF mass spectrometer (Waters) coupled to an Acquity nanochromatography system (Waters). The resulting output files were used to search against a protein database derived from the Erwinia carotovora subsp. atroseptica (Eca) SCRI1043 genome sequence (http://www.sanger.ac.uk; Bell et al., 2004) using MASCOT version 2.1.6 (Matrix Science), to allow identification of the protein species present in each spot.

\section{RESULTS}

\section{Isolation and characterization of spontaneous streptomycin-resistant Ecc mutants}

During a screen for Ecc transposon mutants exhibiting either increased or decreased levels of Car antibiotic production, two streptomycin-resistant derivatives of the wild-type (ATTn10) were isolated which showed reduced Car production (AB1 and AB2; Tables 1 and 2). However, further analysis suggested that they did not carry a transposon insertion (data not shown). We hypothesized that these isolates were spontaneous streptomycin-resistant mutants, and we considered the possibility that the effect on Car production may be related to the streptomycin resistance phenotype. To investigate this further, 15 more streptomycin-resistant derivatives of MS1 (itself a $\mathrm{Lac}^{-}$ derivative of ATTn10), strains M1-M4 and M6-M16 (Tables 1 and 2) were isolated, by plating onto medium containing streptomycin. Spontaneous streptomycin-resistant mutants were very rare, but could be isolated at a frequency of approximately $10^{-13}$ (data not shown).
Streptomycin is an aminoglycoside antibiotic that acts by binding to the bacterial ribosome and causing mistranslation of proteins (reviewed by Kornder, 2002). Spontaneous streptomycin resistance is therefore often associated with mutations in ribosomal protein subunits or rRNA which prevent the antibiotic from binding to the ribosome. Common examples of these mutations include substitutions of lysine- 43 or lysine- 88 of the $S 12$ ribosomal protein subunit, which is encoded by the $r p s L$ gene (e.g. Nair et al., 1993; Shima et al., 1996; Sreevatsan et al., 1996; Björkman et al., 1998; Gregory et al., 2001). In addition, a recent crystal structure study by Carter et al. (2000) showed that streptomycin makes contact with the ribosomal protein S12 when bound to the 30S ribosome of Thermus thermophilus.

To investigate the basis of streptomycin resistance in the Ecc isolates, the rpsL loci were PCR amplified and sequenced using primers AB454 and AB455 (Table 3). Each of the streptomycin-resistant isolates carried a single, point mutation in the rpsL gene which would result in an amino acid change at codon 43, from the Ecc wild-type lysine to either threonine, asparagine or arginine (Table 2). The MICs of streptomycin for mutant strains harbouring the K43N, K43T or K43R mutations (strains M1, M3 and M7 respectively) were greater than $512 \mu \mathrm{g}$ streptomycin $\mathrm{ml}^{-1}$, whereas the MIC of streptomycin for the wildtype MS1 is $16 \mu \mathrm{g} \mathrm{ml}^{-1}$ (data not shown). However, as expected, these strains exhibited little or no cross-resistance to spectinomycin, gentamicin or kanamycin (data not shown).

\section{Antibiotic production by rpsL mutants}

The effects of the rpsL mutations on Car production in overnight cultures were assessed and two phenotypic groups of $r p s L$ mutants were identified: group 1 mutants did not produce any detectable Car, while group 2 mutants produced reduced amounts of Car compared to the wildtype (Fig. 1a). These Car phenotypes could generally be correlated with the nature of the ribosomal protein S12

Table 3. Oligonucleotide primers used in this study

\begin{tabular}{|lccl|}
\hline Primer & Sequence $\left(\mathbf{5}^{\prime} \mathbf{-} \mathbf{3}^{\prime}\right)$ & Annealing temp. & \multicolumn{1}{c|}{ Description } \\
\hline AB454 & CTAGAATTCTGCGTCCTC & $47{ }^{\circ} \mathrm{C}$ & Designed to anneal approx. 100 bp upstream of Eca rpsL ORF \\
AB455 & CATGGAAATACTCCGTTG & $47{ }^{\circ} \mathrm{C}$ & Designed to anneal approx. 100 bp downstream of Eca rpsL ORF \\
\hline
\end{tabular}


mutation: group 1 mutants generally carried $\mathrm{K} 43 \mathrm{~N}$ or K43T mutations, while group 2 strains carried the K43R mutation (Fig. 1a, Table 2). The exception was strain M14, which did not produce Car despite having the group 2 $\mathrm{K} 43 \mathrm{R}$ mutation. It is possible that this strain carries another uncharacterized mutation that affects Car production. Similar results were also seen when filter-sterilized culture supernatant samples, taken throughout growth of representative $\mathrm{K} 43 \mathrm{~N}, \mathrm{~K} 43 \mathrm{~T}$ and $\mathrm{K} 43 \mathrm{R}$ mutants, were assayed for the presence of the Car antibiotic (Fig. 1b).

Since $r p s L$ encodes the ribosomal protein S12, it is likely that any phenotypes associated with $r p s L$ mutations are due to altered translation of one or more cellular proteins. To (a)


Fig. 1. Car antibiotic production and carA transcription by rpsL mutants. (a) Cultures of the strains to be tested were spotted as indicated onto a top agar layer seeded with the E. coli $\beta$-lactam-sensitive strain ESS. Car antibiotic production is indicated by a zone of antibiosis of the E. coli lawn. (b) Car antibiotic present in filter-sterilized culture supernatants was assayed at hourly intervals throughout growth of Ecc rpsL mutants K43N, K43T and $\mathrm{K} 43 \mathrm{R}$, compared to the wild-type, $\mathrm{K} 43$ (strains M1, M3, M7 and MS1 respectively). The culture supernatants were placed in cup wells cut into a top agar layer seeded with $E$. coli strain ESS. The radii of the antibiosis haloes were measured and the data shown are the means of three independent experiments; error bars represent one standard deviation. (c) Transcription of the first gene in the car biosynthetic operon, $\operatorname{car} A$, was followed using a carA:: lac $Z$ transcriptional reporter in the K43N, K43T, K43R and K43 (WT) rpsL genetic backgrounds. Samples were taken hourly throughout growth and $\beta$-galactosidase activity determined. Data shown are the means of three independent experiments; error bars represent one standard deviation. 
test whether a Car regulator was involved in mediating the effect of the rpsL mutations on Car antibiotic production, the effect of the rpsL mutations on transcription of the first gene in the car biosynthetic operon, carA, was investigated. The chromosomal carA::lacZ transcriptional reporter fusion from strain GB7 (Table 1) was moved into the $\mathrm{K} 43 \mathrm{~N}, \mathrm{~K} 43 \mathrm{~T}$ and K43R genetic backgrounds (strains M1, M3 and M7 respectively) by generalized transduction. The $\beta$-galactosidase activities of the resulting strains (7M1, 7M3 and 7M7; Table 1), measured throughout growth, showed that carA transcription was reduced in each $r p s L$ mutant background (Fig. 1c). Transcription of carA was affected most in the $\mathrm{K} 43 \mathrm{~N}$ and $\mathrm{K} 43 \mathrm{~T}$ backgrounds, which correlates well with the effects of the rpsL mutations on Car antibiotic production (Fig. 1a, b, c).

\section{Effect of rpsL mutations on Car regulators}

The effect of the rpsL mutations on transcription of the known Car transcriptional regulators (carI, carR and hor) was assessed. The chromosomal carI: : lacZ and carR:: lacZ transcriptional fusions were introduced into strains $M 1$, M3 and M7 by generalized transduction, using the markers from strains PRTX1 and GB3 respectively (Table 1). Spontaneous streptomycin-resistant derivatives of the hor:: lacZ fusion strain (DET107) were isolated after plating DET107 onto medium containing streptomycin, and the natures of the rpsL mutations were checked by PCR amplification and sequencing (Table 1). Transcription of carI, carR and hor was assessed by assaying $\beta$-galactosidase activity throughout growth. However, no significant differences in transcription of carl, carR or hor were observed in any of the rpsL mutants when compared with strains harbouring the wild-type copy of rpsL (data not shown).

The concentration of the QS signalling molecule 3-oxo-C6HSL was also measured in samples of culture supernatants taken from representatives of each type of rpsL mutant; however, no significant differences were observed in the $r p s L$ mutants compared to the wild-type (data not shown). This suggests that translation of CarI (the 3-oxo-C6-HSL synthase) is unaffected in the rpsL mutant backgrounds.

\section{Addition of the QS signal, 3-0xo-C6-HSL, bypasses the rpsL phenotype}

Interestingly, although $r p s L$ mutants produced apparently wild-type levels of the QS signal 3-oxo-C6-HSL, Car production by these mutants was found to increase when an excess of 3-oxo-C6-HSL was provided (Fig. 2a). In addition, a Western blot analysis showed that CarA protein was undetectable in a K43T mutant in the absence of exogenously added 3-oxo-C6-HSL, but was detectable when an excess of 3-oxo-C6-HSL was present (Fig. 2b). Thus the effects of the K43N and K43T mutations on CarA protein levels and antibiotic production could be partially overcome by provision of an excess of 3-oxo-C6-HSL.

\section{Ecc rpsL mutations have a pleiotropic effect on exoenzyme production and on virulence in planta}

The effects of $r p s L$ mutations on other phenotypes were tested. The K43T and K43N mutants were pleiotropic, exhibiting reduced production of the major exoenzymes of $E c c$ (pectate lyase, cellulase and protease; Fig. 3a) and reduced virulence in potato tuber assays (Fig. 3b). By contrast, the K43R mutant was not significantly affected in either exoenzyme production or virulence (Fig. 3a, b).

\section{Proteomic analysis of the $\mathrm{K} 43 \mathrm{~N}$ ribosomal protein S12 mutant}

To determine whether an rpsL mutation simply affects levels of all proteins within the cell, or affects a specific subset of proteins, a quantitative proteomic analysis was carried out. Intracellular proteins were prepared from

(a)

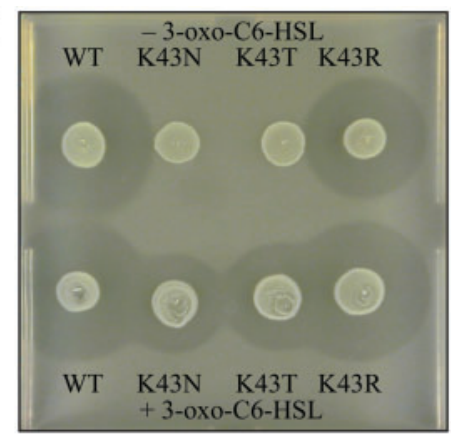

(b)



Fig. 2. The $r p s L$ phenotype can be bypassed by the addition of excess 3-oxo-C6-HSL. (a) Car production by Ecc strains MS1 (K43), M1 (K43N), M3 (K43T) and M7 (K43R) was tested by spotting cultures onto a top agar layer seeded with the $\beta$-lactamsensitive E. coli strain ESS. Drops $(1 \mu \mathrm{l})$ of a $1 \mathrm{mg} \mathrm{ml}^{-1}$ solution of synthetic 3-oxo-C6-HSL were spotted on top of the dried-in culture as indicated. Car production was indicated by antibiosis of the E. coli lawn. (b) Immunodetection of CarA protein. Strains ATTn10 or AB1 (K43T) were cultured in LB medium with or without $1 \mathrm{\mu g} \mathrm{ml}^{-1}$ synthetic 3-oxo-C6-HSL, to the late-exponential phase of growth before harvest. Equivalent amounts of total protein from each strain were run on a $12 \%$ SDS-PAGE gel and then electro-transferred to a PVDF membrane. CarA protein was detected using an anti-CarA antibody. As a control, the antiCarA antibody failed to hybridize to proteins extracted from a carA deletion strain, SM1 (data not presented). 
(a)

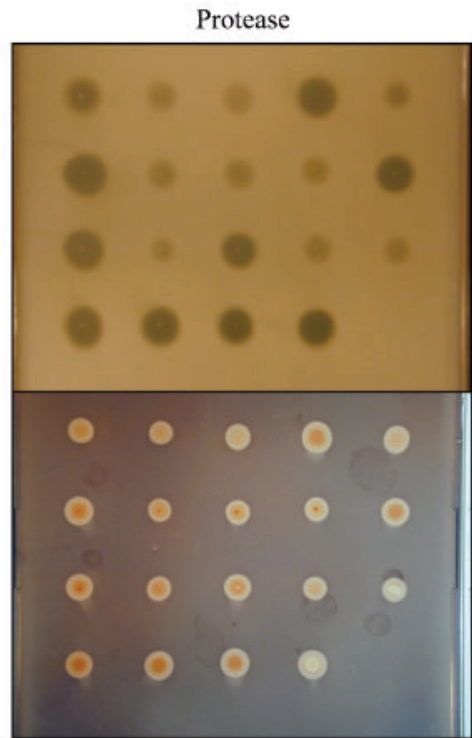

Cellulase
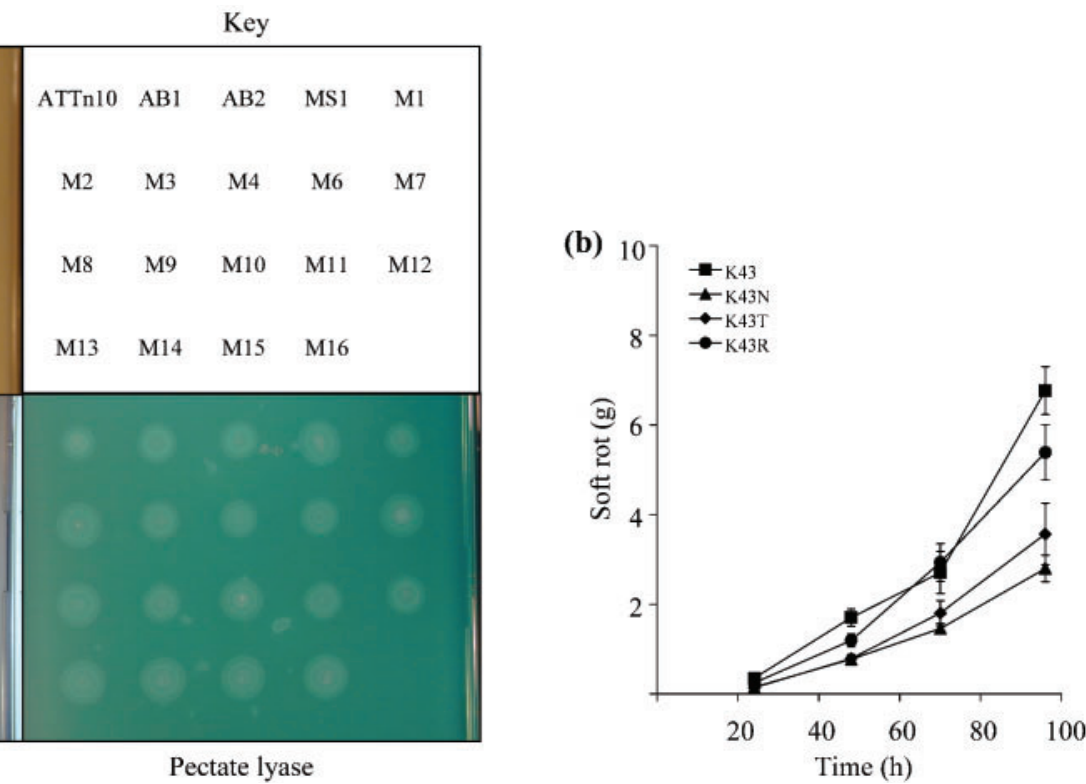

Fig. 3. Effects of $r p s L$ mutations on virulence. (a) Exoenzyme production by $r p s L$ mutants. Aliquots (3 $\mu$ ) of overnight cultures, normalized to an $\mathrm{OD}_{600}$ of 1.0 with fresh LB medium, were spotted as indicated on the relevant assay plates. Cellulase activity is indicated by an orange-red halo on a dark blue background; pectate lyase activity is shown by a double, cream halo on a bluegreen background; protease activity is indicated by a clear halo on an opaque white background. (b) Virulence in potato tuber assays. Potato tubers were inoculated with approx $10^{4}$ c.f.u. of the strains to be tested and incubated at $25^{\circ} \mathrm{C}$. Potatoes were harvested and the mass of soft-rotted tissue was determined at each time point. Results shown are the means of at least eight replicates for each time point; error bars represent one standard error of the mean.

stationary-phase cultures of the wild-type (MS1) and a K43T ribosomal protein S12 mutant (M3), and the intracellular proteomes were subjected to a biological variance analysis (BVA). Representative images are shown in Fig. 4. A total of 55 protein spots were identified that were more abundant in the rpsL mutant compared to the wild-type (circled in Fig. 4a; $>1.5$-fold difference, $P<0.01$ ), while 45 protein spots were less abundant in the rpsL mutant compared to the wild-type (circled in Fig. 4b; $>1.5$-fold difference, $P<0.01$ ). Of these 100 spots, 16 were sufficiently abundant for mass spectrometry analysis (numbered in Fig. 4a and b). Most of these spots contained more than one protein species, so it was not possible to determine unequivocally which species was affected in the $r p s L$ mutant. However, it was possible to identify spot no. 1, which was highly similar to ATP phosphoribosyltransferase from Eca strain SCRI1043. This protein showed an increased abundance in the rpsL mutant. ATP phosphoribosyltransferase catalyses the first step in histidine biosynthesis, the formation of N-1-(5'-phosphoribosyl)ATP from ATP and 5-phosphoribosyl 1-pyrophosphate (Ames et al., 1961; Voll et al., 1967). Spots 12 and 13 were identified as CarB from ATTn10, and spot 15 as CarC from ATTn10. These three protein species were all reduced in abundance in the rpsL mutant compared to the wild-type, which is consistent with the Car phenotype of the rpsL mutant.

\section{DISCUSSION}

In this study, 17 'spontaneous' streptomycin-resistant derivatives of Ecc strain ATTn10 were isolated, carrying a single point mutation within the rpsL gene. These point mutations were predicted to cause an amino acid substitution at codon 43, from the wild-type lysine, to threonine, asparagine or arginine. The MIC of streptomycin for representative K43T, K43N and K43R mutants was determined to be $>512 \mu \mathrm{g} \mathrm{ml}^{-1}$ in each case, compared to the wild-type MIC of $16 \mu \mathrm{g}$ streptomycin $\mathrm{ml}^{-1}$.

The K43R mutants exhibited reduced Car production and slightly reduced virulence compared to the wild-type strains. By contrast, the K43T and K43N mutants produced no detectable Car, reduced amounts of the exoenzymes pectate lyase, cellulase and protease, and were reduced in virulence in potato tuber assays. The effects of the rpsL mutations on Car production were shown to be due to reduced transcription of the car biosynthetic genes. In contrast to our results, other studies involving several Streptomyces species have associated mutations in $r p s L$ with increases in the amount of antibiotic produced (e.g. Shima et al., 1996; Okamoto-Hosoya et al., 2003a, b; Hu \& Ochi, 2001). In Streptomyces coelicolor A3(2), a K88E rpsL mutant exhibited increased actinorhodin production (in addition to streptomycin resistance) due to an increase in protein synthesis caused by upregulation of expression of the 
(a)

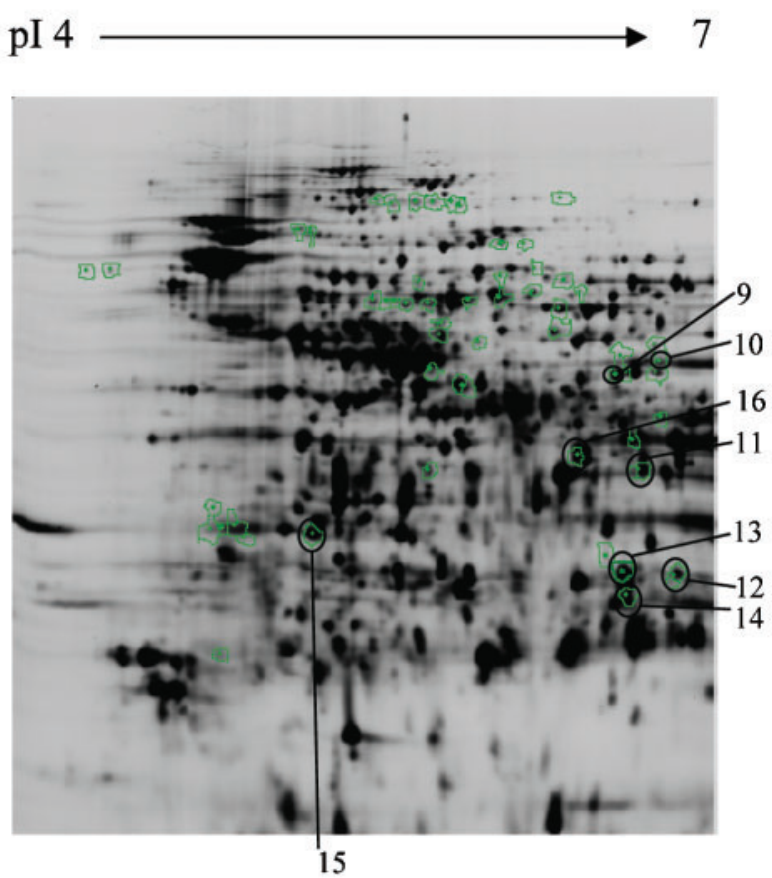

(b)

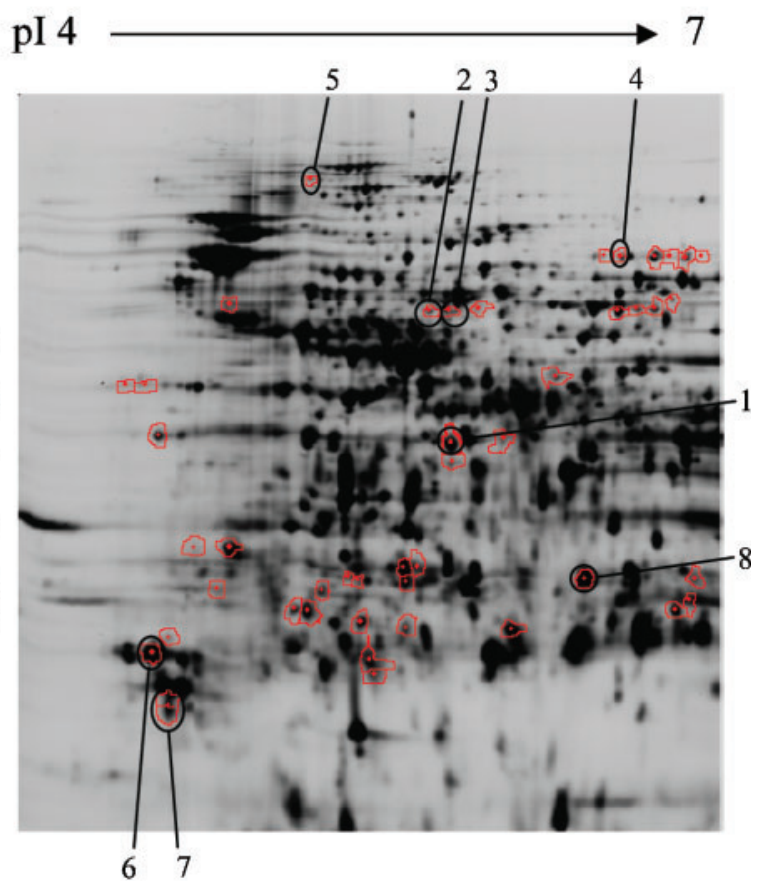

Fig. 4. Intracellular proteomic analysis of an Ecc K43T ribosomal protein $\mathrm{S} 12$ mutant. Results are shown for a representative experiment in which proteins originating from the wild-type were labelled with Cy5 and proteins originating from the K43T mutant were labelled with Cy3. (a) Cy5 image of 2D-DiGE gel with protein spots which are decreased in abundance in the rps $L$ mutant circled in green. The circled and numbered protein spots (9-16) were excised from the gel and analysed by mass spectrometry. (b) Су3 image of 2D-DiGE gel with protein spots which are increased in abundance in the rps $L$ mutant circled in red. The circled and numbered protein spots $(1-8)$ were excised from the gel and analysed by mass spectrometry.

ribosome recycling factor (Okamoto-Hosoya et al., 2003a; Hosaka et al., 2006). K43N, K43T and K43R mutations resulted in streptomycin resistance, but had no impact on antibiotic production in S. coelicolor (Okamoto-Hosoya et al., 2003a). A recent study by Kurosawa et al. (2006) showed that amylase and protease production in Bacillus subtilis strain 168 was enhanced in rpsL K56R mutants (equivalent to K43R of E. coli or Ecc), but production was unaffected in $\mathrm{K} 56 \mathrm{~T}$ and $\mathrm{K} 56 \mathrm{~N}$ mutants. In a study by Björkman et al. (1998), Salmonella rpsL mutations were shown to have an effect on virulence in a mouse model. $\mathrm{K} 43 \mathrm{~T}$ and $\mathrm{K} 43 \mathrm{~N}$ mutants were less able to compete with wild-type Salmonella typhimurium strain LT2 in the mouse model. However, K43R mutants were able to compete effectively with the wild-type LT2.

Since $r p s L$ encodes the ribosomal protein S12, it is possible that translation of one or more proteins is directly affected in the $r p s L$ mutants and the effect on carA transcription may be an indirect one, potentially due to a reduction in translation of a Car regulatory protein. Three Car regulators are known to date: CarI (the 3-oxo-C6-HSL synthase), CarR (the LuxR homologue which responds to the 3-oxo-C6-HSL signal) and Hor (a SlyA family global regulator). Transcription of the genes encoding each of these three proteins appeared unaffected in the $r p s L$ mutants, although it is formally possible that translation of the corresponding messages could be altered. We have shown that production of the 3-oxo-C6-HSL QS signalling molecule is not significantly affected in the rpsL mutant background under the conditions tested, which strongly suggests that the rpsL mutations do not affect CarI enzyme synthesis. It is interesting that the Car phenotypes of $\mathrm{K} 43 \mathrm{~N}$ and K43T rpsL mutants can be partially bypassed by the addition of 3-oxo-C6-HSL. The Car and exoenzyme phenotypes of hor mutants have also been shown to be bypassed by a physiological excess of 3-oxo-C6-HSL (McGowan et al., 2005). Therefore, it is an attractive hypothesis that Hor protein levels may be altered in these rpsL mutants, as this could account for the observed Car, exoenzyme and virulence phenotypes. However, it has not been possible to verify this by Western blot analysis, due to a lack of an anti-Hor antibody with sufficient specificity for Hor (data not shown).

Another possibility was that $r p s L$ mutations might simply result in a global reduction in translation of all cellular proteins. The rpsL mutation did not adversely affect growth rate under the conditions tested (for example, see Fig. 1), which argues against a widespread effect on the intracel- 
lular proteome. However, in order to investigate the effects of the K43T mutation further, we performed a quantitative proteomic comparison of all intracellular proteins extracted from the K43T mutant compared to the wildtype. The results indicated that the abundance of a small subset of intracellular proteins is affected by the K43T mutation: 55 protein spots were increased in abundance in the rpsL mutant, while 45 protein spots were decreased in abundance (Fig. 4). The rpsL mutations therefore affect a subset of the intracellular proteome. It is possible that the protein species affected by these $r p s L$ mutants may share common characteristics, such as their amino acid composition, or share similarities in the codon usage of the corresponding transcripts. This would be an interesting area for further investigation. Three of the protein species that were reduced in the rpsL mutant were identified as CarB or CarC, which is consistent with the effect of the $r p s L$ mutation on carbapenem antibiotic production in Ecc. It is not known whether any of the other proteins that are modulated in the rpsL mutants play a regulatory role in the multiple phenotypes exhibited by the mutant. However, given that regulatory proteins are usually present only in low abundance, it is more likely that these up- or downmodulated proteins identified in the proteomic analysis of the rpsL mutant represent additional targets within the regulon influenced pleiotropically by ribosomal protein S12.

\section{ACKNOWLEDGEMENTS}

We thank Sarah Coulthurst for assistance with the proteomic analysis, as well as the Cambridge Centre for Proteomics, particularly Svenja Hester and Julie Howard. DNA sequencing was carried out by the DNA Sequencing Facility, Department of Biochemistry, University of Cambridge. We thank Lauren Cameron (née Porter) for the polyclonal anti-CarA rabbit serum, Ian Foulds for technical support, and members of the Salmond and Welch groups for constructive discussions. This work was funded by the BBSRC, UK, and through an MRC, UK, studentship awarded to N. J.L.S.

\section{REFERENCES}

Ames, B. N., Martin, R. G. \& Garry, B. J. (1961). The first step of histidine biosynthesis. J Biol Chem 236, 2019-2026.

Bainton, N. J., Stead, P., Chhabra, S. R., Bycroft, B. W., Salmond, G. P. C., Stewart, G. S. A. B. \& Williams, P. (1992a). N-(3Oxohexanoyl)-L-homoserine lactone regulates carbapenem antibiotic production in Erwinia carotovora. Biochem J 288, 997-1004.

Bainton, N. J., Bycroft, B. W., Chhabra, S. R., Stead, P., Gledhill, L., Hill, P. J., Rees, C. E. D., Winson, M. K., Salmond, G. P. C. \& other authors (1992b). A general role for the lux autoinducer in bacterial cell signalling: control of antibiotic synthesis in Erwinia. Gene 116, 87-91.

Barnard, A. M. L. \& Salmond, G. P. C. (2005). Quorum sensing: the complexities of chemical communication between bacteria. Complexus 2, 87-101.

Bell, K. S., Sebaihia, M., Pritchard, L., Holden, M. T., Hyman, L. J., Holeva, M. C., Thomson, N. R., Bentley, S. D., Churcher, L. J. \& other authors (2004). Genome sequence of the enterobacterial phytopatho- gen Erwinia carotovora subsp. atroseptica and characterization of virulence factors. Proc Natl Acad Sci U S A 101, 11105-11110.

Björkman, J., Hughes, D. \& Andersson, D. I. (1998). Virulence of antibiotic-resistant Salmonella typhimurium. Proc Natl Acad Sci U S A 95, 3949-3953.

Burr, T., Barnard, A. M. L., Corbett, M. J., Pemberton, C. L., Simpson, N. J. L. \& Salmond, G. P. C. (2006). Identification of the central quorum sensing regulator of virulence in the enteric phytopathogen, Erwinia carotovora: the VirR repressor. Mol Microbiol 59, 113-125.

Carter, A. P., Clemons, W. M., Brodersen, D. E., Morgan-Warren, R. J., Wimberly, B. T. \& Ramakrishnan, V. (2000). Functional insights from the structure of the $30 \mathrm{~S}$ ribosomal subunit and its interactions with antibiotics. Nature 407, 340-348.

Coulthurst, S. J., Lilley, K. S. \& Salmond, G. P. C. (2006). Genetic and proteomic analysis of the role of $l u x S$ in the enteric phytopathogen, Erwinia carotovora. Mol Plant Pathol 7, 31-45.

Cox, A. R., Thomson, N. R., Bycroft, B. W., Stewart, G. S., Williams, P. \& Salmond, G. P. (1998). A pheromone-independent CarR protein controls carbapenem antibiotic synthesis in the opportunistic human pathogen Serratia marcescens. Microbiology 144, 201-209.

Gregory, S. T., Cate, J. H. \& Dahlberg, A. E. (2001). Streptomycinresistant and streptomycin-dependent mutants of the extreme thermophile Thermus thermophilus. J Mol Biol 309, 333-338.

Holden, M. T. G., McGowan, S. J., Bycroft, B. W., Stewart, G. S. A. B., Williams, P. \& Salmond, G. P. C. (1998). Cryptic carbapenem antibiotic production genes are widespread in Erwinia carotovora: facile trans activation by the carR transcriptional regulator. Microbiology 144, 1495-1508.

Hosaka, T., Xu, J. \& Ochi, K. (2006). Increased expression of ribosome recycling factor is responsible for the enhanced protein synthesis during the late growth phase in an antibiotic-overproducing Streptomyces coelicolor ribosomal rpsL mutant. Mol Microbiol 61, 883-897.

Hu, H. \& Ochi, K. (2001). Novel approach for improving the productivity of antibiotic-producing strains by inducing combined resistant mutations. Appl Environ Microbiol 67, 1885-1892.

Jones, S. J., Yu, B., Bainton, N. J., Birdsall, M., Bycroft, B. W., Chhabra, S. R., Cox, A. R. J., Golby, P., Reeves, P. J. \& other authors (1993). The lux autoinducer regulates the production of exoenzyme virulence determinants in Erwinia carotovora and Pseudomonas aeruginosa. EMBO J 12, 2477-2482.

Kornder, J. D. (2002). Streptomycin revisited: molecular action in the microbial cell. Med Hypotheses 58, 34-46.

Kurosawa, K., Hosaka, T., Tamehiro, N., Inaoka, T. \& Ochi, K. (2006). Improvement of $\alpha$-amlyase production by modulation of ribosomal component protein S12 in Bacillus subtilis 168. Appl Environ Microbiol 72, 71-77.

Laemmli, U. K. (1970). Cleavage of structural proteins during the assembly of the head of bacteriophage T4. Nature 227, 680-685.

McGowan, S. J., Sebaihia, M., Jones, S., Yu, B., Bainton, N., Chan, P. F., Bycroft, B., Stewart, G. S. A. B., Williams, P. \& Salmond, G. P. C. (1995). Carbapenem antibiotic production in Ewinia carotovora is regulated by CarR, a homologue of the LuxR transcriptional activator. Microbiology 141, 541-550.

McGowan, S. J., Sebaihia, M., Porter, L. E., Stewart, G. S. A. B., Williams, P., Bycroft, B. W. \& Salmond, G. P. C. (1996). Analysis of bacterial carbapenem antibiotic production genes reveals a novel $\beta$-lactam biosynthesis pathway. Mol Microbiol 22, 415-426.

McGowan, S. J., Sebaihia, M., O'Leary, S., Hardie, K. R., Williams, P., Stewart, G. S. A. B., Bycroft, B. W. \& Salmond, G. P. C. (1997). Analysis of the carbapenem gene cluster of Erwinia carotovora: definition of the antibiotic biosynthetic genes and evidence for 
a novel $\beta$-lactam resistance mechanism. Mol Microbiol 26, 545556.

McGowan, S. J., Barnard, A. M. L., Bosgelmez, G., Sebaihia, M., Simpson, N. J. L., Thomson, N. R., Todd, D. E., Welch, M., Whitehead, N. A. \& Salmond, G. P. C. (2005). Carbapenem antibiotic biosynthesis in Erwinia carotovora is regulated by physiological and genetic factors modulating the quorum sensing-dependent control pathway. Mol Microbiol 55, 526-545.

Nair, J., Rouse, D. A., Bai, G.-H. \& Morris, S. L. (1993). The $r p s L$ gene and streptomcyin resistance in single and multiple drugresistant strains of Mycobacterium tuberculosis. Mol Microbiol 10, 521-527.

Okamoto-Hosoya, Y., Hosaka, T. \& Ochi, K. (2003a). An aberrant protein synthesis activity is linked with antibiotic overproduction in rpsL mutants of Streptomyces coelicolor A3(2). Microbiology 149, 32993309.

Okamoto-Hosoya, Y., Okamoto, S. \& Ochi, K. (2003b). Development of antibiotic-overproducing strains by site-directed mutagenesis of the rpsL gene in Streptomyces lividans. Appl Environ Microbiol 69, 4256-4259.

Rivet, M. M. (1998). Investigation into the regulation of exoenzyme production in Erwinia carotovora subspecies carotovora. $\mathrm{PhD}$ thesis, University of Warwick, UK.

Sambrook, J., Fritsch, E. F. \& Maniatis, T. (1989). Molecular Cloning: a Laboratory Manual, 2nd edn. Cold Spring Harbor, NY: Cold Spring Harbor Laboratory.

Shima, J., Hesketh, A., Okamoto, S., Kawamoto, S. \& Ochi, K. (1996). Induction of actinorhodin production by $r p s L$ (encoding ribosomal protein S12) mutations that confer streptomycin resistance in Streptomyces lividans and Streptomyces coelicolor A3(2). J Bacteriol 178, 7276-7284.

Sreevatsan, S., Pan, X., Stockbauer, K. E., Williams, D. L., Kreiswirth, B. N. \& Musser, J. M. (1996). Characterization of rpsL and rrs mutants in streptomycin-resistant Mycobacterium tuberculosis isolates from diverse geographic localities. Antimicrob Agents Chemother 40, 10241026.

Thomson, N. R., Cox, A., Bycroft, B. W., Stewart, G. S. A. B., Williams, P. \& Salmond, G. P. C. (1997). The Rap and Hor proteins of Erwinia, Serratia and Yersinia: a novel subgroup in a growing superfamily of proteins regulating diverse physiological processes in bacterial pathogens. Mol Microbiol 26, 531-544.

Toth, I. K. \& Birch, P. R. J. (2005). Rotting softly and stealthily. Curr Opin Plant Biol 8, 424-429.

Toth, I., Perombelon, M. \& Salmond, G. (1993). Bacteriophage $\phi K \mathrm{~K}$ mediated generalized transduction in Erwinia carotovora subspecies carotovora. J Gen Microbiol 139, 2705-2709.

Voll, M. J., Appella, E. \& Martin, R. G. (1967). Purification and composition studies of phosphoribosyladenosine triphosphate: pyrophosphate phosphoribosyltransferase, the first enzyme of histidine biosynthesis. J Biol Chem 242, 1760-1767.

Waters, C. M. \& Bassler, B. L. (2005). Quorum sensing: cell-to-cell communication in bacteria. Annu Rev Cell Dev Biol 21, 319346.

Whitehead, N. A., Barnard, A. M. L., Slater, H., Simpson, N. J. L. \& Salmond, G. P. C. (2001). Quorum-sensing in Gram-negative bacteria. FEMS Microbiol Rev 25, 365-404.

Edited by: W. J. Quax 